\title{
The spectrum of $\beta$ thalassaemia mutations in the UAE national population
}

\author{
R Quaife, L Al-Gazali, S Abbes, P Fitzgerald, A Fitches, D Valler, J M Old
}

\begin{abstract}
The $\beta$ thalassaemia alleles in $50 \beta$ thalassaemia heterozygotes originating from many parts of the United Arab Emirates (UAE) have been characterised using the allele specific priming technique of the polymerase chain reaction (PCR). The IVSI-5 $(\mathbf{G} \rightarrow \mathbf{C})$ mutation was found to be present in $66 \%$, while six other alleles occurred at the much lower frequencies of $2 \%$ to $8 \%$. These were codon $8 / 9(+G)$, IVSI-1, $3^{\prime}$ end $(-25 \mathrm{bp})$, codon 5 ( $\left.-\mathrm{CT}\right)$, IVSII-1 $(\mathbf{G} \rightarrow \mathbf{A})$, codon $30(\mathbf{G} \rightarrow \mathbf{C})$, and codon $15(G \rightarrow A)$. The mutation types and percentages are compared with other Mediterranean Arab countries and neighbouring areas. It is proposed that IVSI-5 and other Asian Indian mutations were introduced into the UAE by population migration from the region previously known as Baluchistan. These findings should be useful for genetic counselling and the development of a first trimester prenatal diagnosis programme based on direct detection of mutations in the UAE.
\end{abstract}

(f Med Genet 1994;31:59-61)

$\beta$ thalassaemia is a highly heterogeneous group of inherited disorders of $\beta$ globin gene expression, ${ }^{1}$ and more than 140 different $\beta$ globin gene mutations have been identified in the world. ${ }^{2}$ The population of the United Arab Emirates (UAE) is diverse, consisting of a total of about 2 to 2.5 million people. Fewer than $20 \%$ are UAE nationals, ${ }^{3}$ but within this small group a variety of single gene defects occurs, of which $\beta$ thalassaemia is one of the most prevalent. ${ }^{4}$ Because of consanguinity, there are many homozygous $\beta$ thalassaemia subjects who need special follow up and present a real health problem for their families and the community as a whole. In order to make possible a simplified carrier detection and prenatal diagnosis service we have used a non-radioactive PCR technique based on allele specific priming. Here, we report our findings and make a comparison between our data and those observed in neighbouring Asian regions and Arab Mediterranean countries.

\section{Subjects and methods}

SUBJECTS

A total of 101 blood samples was collected from 27 families of UAE nationals consisting of carrier parents and at least one child with $\beta$ thalassaemia major. Over $30 \%$ of these families were known to be consanguineous, the parents being first cousins. Most of our patients came from Al-Ain District, Sharjah, and Ra'sal Khaymah and were referred to the genetic clinic of Al-Jimi and Tawam Hospital (Al-Ain). Furthermore, although not obviously related, over $50 \%$ of the subjects shared the surname Baluchi which is a common surname in the UAE population as a whole. A phenotypic study including haematological data and haemoglobin electrophoresis showed that the majority of patients $(85 \%)$ had $\beta^{+}$thalassaemia.

\section{METHODS}

DNA was extracted from the white blood cells of EDTA anticoagulated blood by the method of Kunkel et al. ${ }^{5}$ The $\beta$ thalassaemia mutations were characterised by a PCR method based on allele specific priming known as the amplication refractory mutation system (ARMS) using the method described by Varawalla et $a l .{ }^{6}$ Each parental DNA sample was screened using the mutation specific primers listed in table 1 until the mutation was identified.

The mutations were also confirmed in each homozygous patient and any heterozygous sibs. The PCR conditions and methods used were as previously described ${ }^{6}$ except that the annealing temperature for the codon $44(-\mathrm{C})$ and IVSII-645 ( $-\mathrm{C}$ ) primers was $60^{\circ} \mathrm{C}$.

\section{Results}

Using the allele specific priming technique we were able to define the molecular defects in 23 families by either screening each parental DNA sample or screening the probands' DNA for two mutations. In four families, one of the parental mutations remained uncharacterised because insufficient or no DNA was obtained from the blood samples to allow completion of the screening procedure for all 22 mutations (table 1). Seven $\beta$ thalassaemia mutations were identified in a total of 50 parental DNA samples, with the most prevalent allele being the $\beta^{+}$IVSI-5 $(\mathrm{G} \rightarrow \mathrm{C})$ mutation (fig 1). This was observed at a frequency of $66 \%$ in contrast to the other six alleles which were found at much lower frequencies, ranging between $8 \%$ and $2 \%$ (table 2 ). These were codon $8 / 9(+G)$, the 25 bp deletion (IVSI, $3^{\prime}$ end), codon 5 $(-C T)$, codon $30(G \rightarrow C)$, and codon 15 $(G \rightarrow A)$. Thirteen of the patients were found to be homozygous for the IVSI-5 mutation, and three patients were homozygous for one of the rarer alleles, namely codon 30 , codon $8 / 9$, and codon 5 . Thus, there were 16 out of 23 families $(70 \%)$ in our sample in which each parent was found to carry the same $\beta$ thalassaemia mutation. 
Table 1 Primer sequences used for the detection of $\beta$ thalassaemia mutations by the allele specific priming technique.

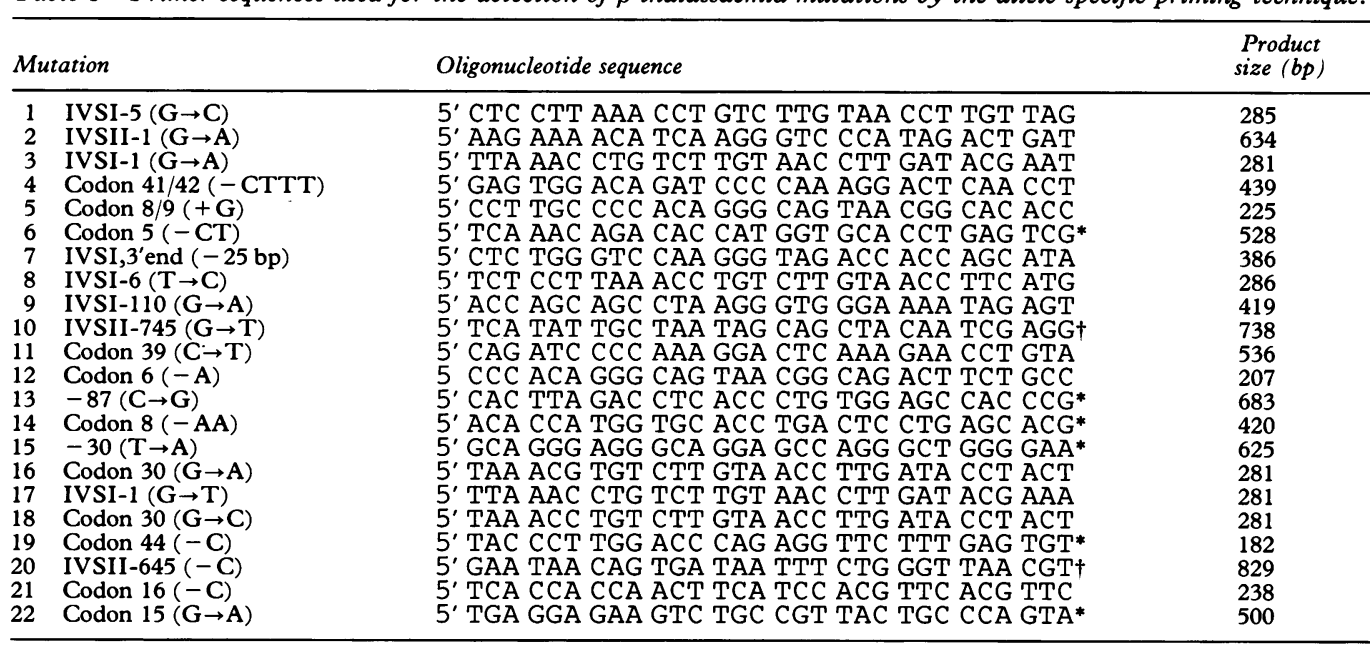

Other primers used: the above primers were coupled with either primer A: $5^{\prime}$-ACC TCA CCC TGT GGA GCCAC; primer B (with primers marked*): 5' CCC CTT CCT ATG ACA TGA ACT TAA; or primer C (with primers marked †): $5^{\prime}$ GAG TCA AGG CTG AGA GAT GCA GGA. The internal control primers used were primer C plus primer D: 5 ' CAA TGT ATC ATG CCT CTT TGC ACC (which amplify a 861 bp frament from the $3^{\prime}$ end of the $\beta$ globin marked + in which case internal control primers E: $5^{\prime}$ AGT GCT GCA AGA AGA ACA ACT ACC and F: $5^{\prime}$ CTC TGC ATC ATG GGC AGT GAG CTC were used (these amplify a 323 bp froment normal $\beta$ globin gene sequence were: for IVSI-5 $(\mathrm{G} \rightarrow \mathrm{C}) 5^{\prime}$ CTC CTT AAA CCT GTC TTG TAA CCT TGT TAC: for codon 8/9 (+G): 5' CCT TGC CCC ACA GGG CAG TAA CGG CAC ACT; for codon 5 (-CT): 5' CAA ACA GAC ACC ATG GTG CAC CTG ACT CCT*: for codon $30(\mathrm{G} \rightarrow \mathrm{C}) 5^{\prime}$ TAA ACC TGT CTT GTA ACC TTG ATA CCT ACC.

\section{Discussion}

This study showed the presence of seven different mutations causing $\beta$ thalassaemia in the UAE population studied. A comparison of the UAE $\beta$ thalassaemia mutation profile with those already defined in areas around the $\mathrm{UAE}^{6-8}$ and from Mediterranean Arab countries $^{9-11}$ showed that none of these profiles was similar to that of the UAE (table 3). In the Mediterranean countries to the west of the UAE the most common mutation found is IVSI-110, with a reported relative frequency ranging from $27 \%$ in Egypt to $62 \%$ in the Lebanon. Further west is codon 39, ranging from $22 \%$ in Greece to $95 \%$ in Sardinia. However, these two mutations were not observed at all in our UAE samples. Although the most frequent mutation in Iran and north-

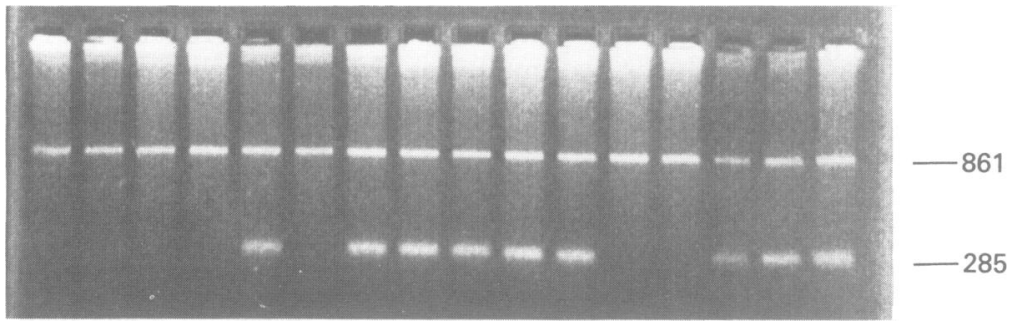

Figure 1 Screening for the mutation at IVS-I nucleotide $5(G \rightarrow C)$. Ethidium bromide stained gel electrophoresis of the amplified products of 16 parental DNA samples with the primer for IVSI-5 $(G \rightarrow C)$ coupled with primer $A$ plus internal control primers $C$ and $D$ (table 1). The $285 \mathrm{bp}$ product seen in nine of the 16 tracks indicates the presence of the mutation.

Table 2 Characterisation and frequencies of the different mutations observed in UAE $\beta$ thalassaemia heterozygotes.

\begin{tabular}{lccl}
\hline Mutation & No of alleles & $\begin{array}{c}\text { Relative frequencies } \\
(\%)\end{array}$ & $\begin{array}{c}\text { Phenotype } \\
\text { (in homozygotes) }\end{array}$ \\
\hline IVS1-5 $(\mathrm{G} \rightarrow \mathrm{C})$ & 33 & 66 & $\beta^{+}$ \\
FR 8/9 $(+\mathrm{G})$ & 4 & 8 & $\beta^{\circ}$ \\
25 bp del & 4 & 8 & $\beta^{\circ}$ \\
Codon 5 $(-\mathrm{CT})$ & 3 & 6 & $\beta^{\circ}$ \\
IVSII-1 $(\mathrm{G} \rightarrow \mathrm{A})$ & 3 & 6 & $\beta^{\circ}$ \\
Codon $30(\mathrm{G} \rightarrow \mathrm{C})$ & 2 & 4 & $\beta^{+}$ \\
Codon $15(\mathrm{G} \rightarrow \mathrm{A})$ & 1 & 2 & $\beta^{\circ}$ \\
\hline
\end{tabular}

west Pakistan has been found to be IVSII-1 $(22 \%)$ and codon $8 / 9(44 \%)$ respectively, the UAE mutation profile has five mutations in common with those found in Iran and three with north-west Pakistan, the latter having the nearest comparable frequency of IVSI-5 $(35 \%)$.

The $\beta^{+}$IVSI-5 $(\mathrm{G}-\mathrm{C})$ mutation is interesting because it has been previously found in the Chinese $\mathrm{e}^{12}$ and Asian Indian populations ${ }^{13}$ but has rarely been reported among Mediterranean Arabs. ${ }^{10} \mathrm{With}$ its high frequency in the UAE population, it may be that this allele was introduced into the UAE by gene flow from the Asian subcontinent. We have noted both in our sample and in the population as a whole that the surname Baluchi is common. Out of $28 \beta$ thalassaemia trait subjects with the surname Baluchi, $24(86 \%)$ were found to carry the IVSI-5 mutation. A map of the areas close to the UAE (fig 2) shows the region previously known as Baluchistan, which is across the Strait of Hormuz and is now in Iran, Afghanistan, and Pakistan. The people of this area, known as Baluchis, have a history going back over 2000 years and occupy one of the most isolated regions in the world. There has always been an exodus of these people mainly to Oman and the UAE. Indeed Frye in $1961^{14}$ drew attention to the earliest inhabitants of Baluchistan who, being defeated by the Iranian Gedrasians sometime before Alexander's conquest, subsequently moved across the Gulf to Arabia.

The close linkage of the $\beta^{+}$IVSI-5 allele with the surname Baluchi and the anthropological information mentioned above suggest that a proportion of the UAE population could be made up of immigrants from the region previously known as Baluchistan. These people might have travelled across the Strait of Hormuz via Oman bringing the $\beta^{+}$IVSI- 5 allele to the UAE. Other mutations present, such as codon $8 / 9,25 \mathrm{bp}$ del, and IVSII-1 may 
Table 3 Molecular heterogeneity of $\beta$ thalassaemia alleles in different populations around the UAE.

\begin{tabular}{|c|c|c|c|c|c|c|c|}
\hline & \multicolumn{3}{|c|}{ Mediterranean areas } & \multirow[b]{2}{*}{ United Arab Emirates } & \multicolumn{3}{|l|}{ Asian areas } \\
\hline & Egypt & Syria & Lebanon & & Saudi Arabia & NW Pakistan & Iran \\
\hline \multirow{3}{*}{$\begin{array}{l}\text { Common } \\
\text { alleles }\end{array}$} & IVSI-110 (27\%) & IVSI- 110 & IVSI-1 $10(62 \%)$ & IVSI-5 (66\%) & IVSI-110 & IVSI-5 (35\%) & IVSII-1 (22\%) \\
\hline & IVSI-6 $(19 \%)$ & - & IVSI-6 $(8 \%)$ & FR $8 / 9(8 \%)$ & IVSII-645 & FR $8 / 9(44 \%)$ & IVS-5 $(13 \%)$ \\
\hline & IVSI-1 (10\%) & IVSI-1 & CD29 (8\%) & 25 bp del $(8 \%)$ & - & CD41/42 (10\%) & IVSI-6 $(9 \%)$ \\
\hline \multirow{5}{*}{$\begin{array}{l}\text { Rare } \\
\text { alleles }\end{array}$} & IVSII-745 & IVSII-745 & IVSII-745 & CD5 & CD6 & CD15 & FR 8/9 \\
\hline & IVSII-1 & - & IVSII-1 & IVSII-1 & IVSII-1 & IVSI-1 & 25 bp del \\
\hline & FR6 & - & CD8 & CD30 & - & - & CD5 \\
\hline & CD37 & - & IVSI-5 & CD15 & - & - & CD16 \\
\hline & CD39 & CD39 & CD39 & - & CD39 & - & CD39 \\
\hline
\end{tabular}

The data in this table came from references $6,7,8,9,10$, and 11. No frequency data was reported in reference 8 (for Saudi Arabia) and reference 11 (for Syria).

also have been introduced by population migration, as these mutations have been observed in families of Iranian, Pakistani, and Omani origin. On the other hand, the origin of the Mediterranean mutations (codon 5 and codon 30) remains obscure and could hardly be explained by population migration of Mediterranean Arabs since these mutations are more prevalent in the UAE than in Mediterranean Arab countries.

In conclusion, the use of the ARMS version of the PCR technique enabled us to characterise the $\beta$ thalassaemia allele in all of our patients for whom sufficient DNA was obtained. Seven different mutations were found, all of which are severe $\beta^{+}$or $\beta^{0}$ types

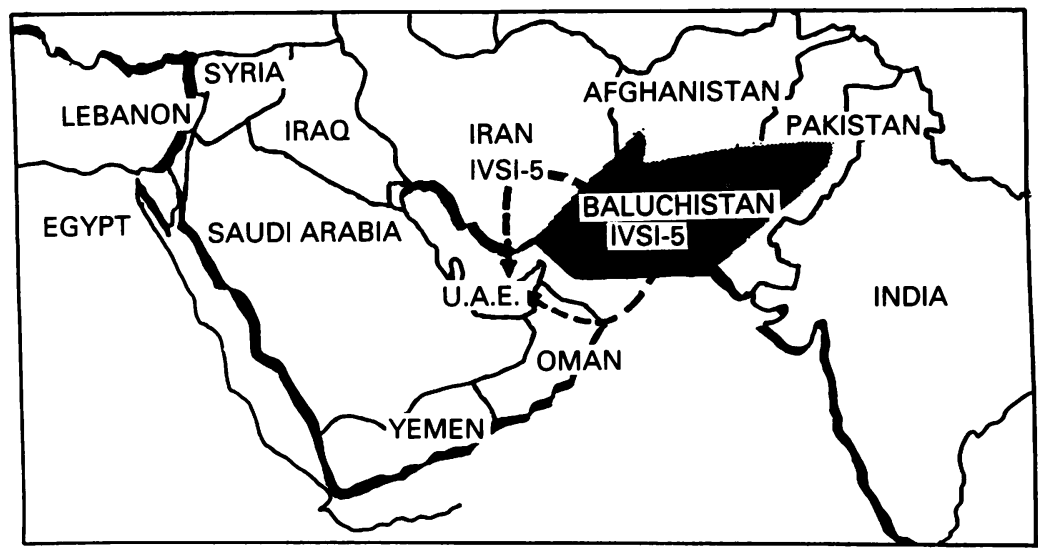

Figure 2 Map showing the location of the region previously known as Baluchistan (shaded area) and the possible introduction of the IVSI-5 $(G \rightarrow C)$ mutation to the $U A E$ by population migration. resulting in the phenotype of $\beta$ thalassaemia major in the homozygous state. These data will be useful for genetic counselling and the preparation of a comprehensive programme of carrier detection and prenatal diagnosis.

1 Weatherall DJ, Clegg JB, eds. The thalassaemia syndromes. Oxford: Blackwell Scientific Publications, 1981

2 Baysal E. The $\beta$ and $\delta$ thalassemia repository. Hemoglobin 1992;16:237 $\beta$ and

3 The Software Toolworks World Atlas. People of the United Arab Emirates. Chatsworth, CA 91311, 1989.

4 White JM, Byrne M, Richards R, Buchanan T, Katsoulis E, Weerasingh $\mathrm{K}$. Red cell genetic abnormalities in Peninsular Arabs: sickle haemoglobin, G6PD deficiency, and a and $\beta$ thalassaemia. $\mathcal{F}$ Med Genet 1986;23:245-51.

5 Kunkel LM, Smith KD, Boyer SH. Analysis of human Y chromosome-specific reiterated DNA in chromosome variants. Proc Natl Acad Sci USA 1977;74:1245-9.

6 Varawalla NY, Old JM, Sarkar R, Venkatesan R, Weatherall DJ. The spectrum of $\beta$ thalassemia mutations on the Indian subcontinent: the basis for prenatal diagnosis. $\mathrm{Br} \mathcal{F}$ Haematol 1991;78:242-7.

7 Varnavides L, Old J, Mitchell M, et al. Proceedings of the 5 th international conference on thalassemias and the haemoglobinopathies, 1993.

8 El-Hazmi MAF, Warsy SA, Al-Favazi. Molecular pathology of $\beta$ thalassemia in Arabia. 4th international conference on thalassemia and the haemoglobinopathies, 1991.

9 Novelletto A, Hafez M, Deidda G, et al. Molecular characterization of $\beta$ thalassemia mutations in Egypt. Hum Genet 1990;85:272-4.

10 Chehab FF, Derkaloustian V, Khouri FP, Deeb SS, Kan $Y W$. The molecular basis of $\beta$ thalassemia in Lebanon: application to prenatal diagnosis. Blood 1987;69:1141-5.

$11 \mathrm{Al}-$ Quobaili F. $\beta$ thalassemia mutations in Syria detected by the polymerase chain reaction. Proceedings of the 5 th international conference on thalassemias and the hemoglobinopathies, 1993 .

12 Cheng TC Orkin SH, Antonarakis SE, Potter MS, Sexton JP. $\beta$ thalassemia in Chinese: use of in vivo RNA analysis and oligonucleotide hybridization in systematic characterization of molecular defects. Proc Natl Acad Sci USA 1984;81:2821.

13 Kazazian HH, Orkin SH, Antonarakis SE, et al. Molecular characterization of $\beta$ thalassemia mutations in Asian

14 Frye RN. Remarks on Baluchi history. Cent Asiatic $\mathfrak{f}$ $1961 ; 6: 44-50$. 